\title{
The NAM/ATAF1/2/CUC2 transcription factor PpNAC.A59 enhances PpERF.A16 expression to promote ethylene biosynthesis during peach fruit ripening
}

Zhi-Hua Guo ${ }^{1}$, You-Jia Zhang ${ }^{1}$, Jia-Long Yao $\mathbb{E}^{2}$, Zhi-Hua Xie ${ }^{1}$, Yu-Yan Zhang ${ }^{3}$, Shao-Ling Zhang ${ }^{1}$ and Chao Gu (1) ${ }^{1 凶}$

\begin{abstract}
Peach is a typical climacteric fruit that releases ethylene during fruit ripening. Several studies have been conducted on the transcriptional regulation of ethylene biosynthesis in peach fruit. Herein, an ethylene response factor, PpERF.A16, which was induced by exogenous ethylene, could enhance ethylene biosynthesis by directly inducing the expression of 1-aminocyclopropane-1-carboxylic acid synthase (PPACS1) and 1-aminocyclopropane-1-carboxylic acid oxidase (PpACO1) genes. Moreover, the NAM/ATAF1/2/CUC2 (NAC) transcription factor (TF) PpNAC.A59 was coexpressed with PpERF.A16 in all tested peach cultivars. Interestingly, PpNAC.A59 can directly interact with the promoter of PpERF.A16 to induce its expression but not enhance LUC activity driven by any promoter of PpACS1 or PpACO1. Thus, PpNAC.A59 can indirectly mediate ethylene biosynthesis via the NAC-ERF signaling cascade to induce the expression of both PPACS1 and PpACO1. These results enrich the genetic network of fruit ripening in peach and provide new insight into the ripening mechanism of other perennial fruits.
\end{abstract}

\section{Introduction}

Fruit ripening is the last stage of the fruit developmental process and is accompanied by a series of physiological and metabolic changes in color, texture, flavor, and nutritional compounds ${ }^{1}$. Fleshy fruits are classically defined as climacteric or nonclimacteric; the former exhibit a burst of ethylene production and respiration during ripening, while the latter do not ${ }^{2}$. Ethylene is a major signaling molecule that controls fruit ripening in climacteric fruits ${ }^{3}$ and plays an important role in the fruit ripening of nonclimacteric fruits ${ }^{4,5}$.

Ethylene biosynthesis and signal transduction have been widely studied in angiosperms ${ }^{6-8}$. Ethylene biosynthesis is

\footnotetext{
Correspondence: Chao Gu (quchao@njau.edu.cn)

'College of Horticulture/State Key Laboratory of Crop Genetics and Germplasm Enhancement, Nanjing Agricultural University, 210095 Nanjing, China

${ }^{2}$ New Zealand Institute of Plant \& Food Research Ltd, Private Bag 92169, Auckland 1142, New Zealand

Full list of author information is available at the end of the article
}

catalyzed by two enzymes, 1-aminocyclopropane-1carboxylic acid synthase (ACS), and oxidase (ACO). Synthesized ethylene activates the expression of ripeningrelated genes through transcription factors (TFs), ETHYLENE INSENSITIVE-like factors and ethylene responsive factors (ERFs). In tomato, LeERF1 promotes fruit ripening ${ }^{9}$, while SlERF6 delays fruit ripening ${ }^{10}$. In perennial fruit trees, MaERF9, MdERF3, and PbERF24 induce ethylene biosynthesis during fruit ripening in banana, apple, and pear, respectively ${ }^{11-13}$. In contrast, MaERF11, MdERF2, and CpERF9 inhibit ethylene biosynthesis during fruit ripening in banana, apple, and papaya, respectively ${ }^{11,12,14}$. Notably, apple MdERF3 is the downstream target of MdERF2, MdMYC2, and MdARF5 ${ }^{12,15,16}$. These findings suggest that ERFs are important in ethylene biosynthesis during fruit ripening.

In addition to ERF, other TFs, such as NAM/ATAF1/2/ CUC2 (NAC), MADS-box, homeobox, apetala 2, SQUAMOSA promoter binding protein (SBP), auxin response

\section{(c) The Author(s) 2021}

(c) (i) Open Access This article is licensed under a Creative Commons Attribution 4.0 International License, which permits use, sharing, adaptation, distribution and reproduction c. in any medium or format, as long as you give appropriate credit to the original author(s) and the source, provide a link to the Creative Commons license, and indicate if changes were made. The images or other third party material in this article are included in the article's Creative Commons license, unless indicated otherwise in a credit line to the material. If material is not included in the article's Creative Commons license and your intended use is not permitted by statutory regulation or exceeds the permitted use, you will need to obtain permission directly from the copyright holder. To view a copy of this license, visit http://creativecommons.org/licenses/by/4.0/. 
factor (ARF), and zinc-finger proteins, are also involved in mediating ethylene biosynthesis in climacteric fruits ${ }^{8,17-20}$. Among the NAC TFs, NOR, SINAC4, and NAC-like 1 are positive regulators of fruit ripening ${ }^{21-23}$, while SINAC1 is a transcriptional repressor of ripening-related genes in tomato $^{24}$. AdNAC6 and AdNAC7 can bind to the AdACS1 and $A d A C O 1$ promoters to stimulate its expression and increase ethylene production in kiwifruit ${ }^{25}$. MaNAC1 and MaNAC2 can directly interact with ethylene signaling components, such as ETHYLENE INSENSITIVE, in banana $^{26}$. CmNAC-NOR is located in a quantitative trait locus for climacteric ripening and is likely associated with ethylene biosynthesis and fruit ripening in $\operatorname{melon}^{27}$. Moreover, a heterodimer of two NACs can activate the transcription of an MYB $\mathrm{TF}^{28}$, indicating that the NAC TF is probably an upstream factor that regulates the expression of downstream $\mathrm{TF}(\mathrm{s})$. These findings suggest that NAC could be involved in regulating ethylene biosynthesis by directly and/or indirectly inducing ethylene biosynthesis genes. This suggestion is supported by previous reports, in which three MADS-box TFs, RIN, FUL1, and FUL2, can mediate the expression of $A C S$ genes and their upstream TFs, including RIN, NOR, and CNR (an SBP TF) ${ }^{29,30}$.

Peach (Prunus persica) emits a large amount of ethylene during fruit ripening ${ }^{31,32}$. Exogenous ethylene accelerates fruit softening and induces the expression of softening-related genes ${ }^{33}$, such as endopolygalacturonase (PpendoPGM) controlling the melting flesh phenotype in the clingstone melting flesh (CMF) peach fruit ${ }^{34}$. Moreover, ethylene biosynthesis and signal transduction in peach can be interrupted by aminoethoxyvinylglycine and 1-methylcyclopropene (1-MCP), respectively, leading to the delay of fruit ripening ${ }^{35,36}$. Therefore, ethylene is crucial for catalyzing fruit ripening in peach. The genes PpACS1 and PpACO1, which encode the two catalytic enzymes for ethylene biosynthesis, have been identified in peach fruit ${ }^{37-39}$. Two TFs, PpHB.G7 and PpTCP.A2, were identified to be involved in peach fruit ripening ${ }^{40,41}$. PpHB.G7 can bind to the PpACS1 and PpACO1 promoters to induce the expression of both genes and increase ethylene production $^{40}$, while PpTCP.A2 can inhibit ethylene biosynthesis by negatively affecting PpACS1 expression ${ }^{41}$. To broaden the transcription regulation network of ethylene biosynthesis in peach, two TFs, PpERF.A16 and PpNAC.A59 were isolated from peach fruit. Of these, PpERF.A16 could directly bind to the PpACS1 and PpACO1 promoters to positively regulate ethylene biosynthesis in peach fruit. PpNAC.A59 was coexpressed with $P p E R F . A 16$, and PpNAC.A59 could directly bind to the promoter of PpERF.A16 to indirectly promote ethylene biosynthesis in peach fruit. This study further elucidates the molecular network of ethyleneinduced fruit ripening in peach and other perennial fruit trees.

\section{Results}

\section{Identification of $A C S, A C O$, and ERF genes during fruit ripening}

Members of the $A C S, A C O$, and ERF gene families were isolated from peach and other fruit trees. A total of 106 $A P 2 / E R F$ genes (Table S1) were detected in the peach genome and could be divided into two groups, $A$ and $B$ (Fig. S1). Of the two groups, group A corresponded to subfamily ERF, which comprised 56 clusters (A1 $\rightarrow$ A56), and group B contained the AP2, RAV, and soloist subfamilies. Moreover, six members of the ACS gene family were detected in peach (Fig. S2) along with 32 possible members of the ACO (or ACO-like) gene family (Fig. S3). Based on the RNA-Seq data of fruit samples of the peach cultivars Nanshantiantao (NS) and Zhaohui $(\mathrm{ZH})$ in a previous study ${ }^{34}$, the $A C S, A C O$, and $A P 2 / E R F$ genes, which had higher levels of expression in ripening fruit than in developing fruit (Fig. 1A and Table S2), were detected. Based on previous reports ${ }^{37,40}$, the ACS gene Ppa004774m was identical to PpACS1, and the two ACO genes, Pp008791m and Ppa009228m, were identical to $P p A C O 1$ and PpACO3, respectively. The three ERF genes belonged to the A16, A29, and A31 clusters and were thus designated PpERF.A16 (Ppa008730m), PpERF.A29 (Ppa021711m), and PpERF.A31.1 (Ppa007193m), respectively (Table S1). However, the expression level of $P p A C O 3$ in ripening fruit was similar to that in developing fruit of cv. Xiahui No. 5 (Fig. 1B) and cvs. Yuhualu, Chumei, and Hujimilu (Fig. 1C). Moreover, PpERF.31.1 and PpERF.A29 had similar levels of expression between developing and ripening fruits of some cultivars (Fig. 1C), although these two genes were more highly expressed in ripening fruit than in developing fruit of $\mathrm{cv}$. Xiahui No.5 (Fig. 1B). These results indicate that PpACO3, PpERF.31.1, and PpERF.A29 are not involved in peach fruit ripening. In contrast, $P p A C S 1, P p A C O 1$, and $P p E R F$. $A 16$ were more highly expressed in ripening fruit than in developing fruit of all 12 tested cultivars (Fig. 1B, C) and thus are probably associated with peach fruit ripening.

\section{Interaction of PpERF.A16 with the promoters of the PpACS1, PpACO1, and PpendoPGM genes}

Similar to the previous study ${ }^{40}$, to test whether PpERF. A16 could bind to the promoters of ripening-related genes, PpACS1, PpACO1, and PpendoPGM, dualluciferase assays were conducted and revealed that the $L U C$ activities that were individually driven by the PpACS1, PpACO1, or PpendoPGM promoters were significantly increased in Arabidopsis protoplasts overexpressing PpERF.A16 (35S::PpERF.A16) compared to Arabidopsis protoplasts transformed with an empty vector or without any transformation (control; Fig. 2B). This result indicates that PpERF.A16 can interact with the promoters of PpACS1, PpACO1, and PpendoPGM. 

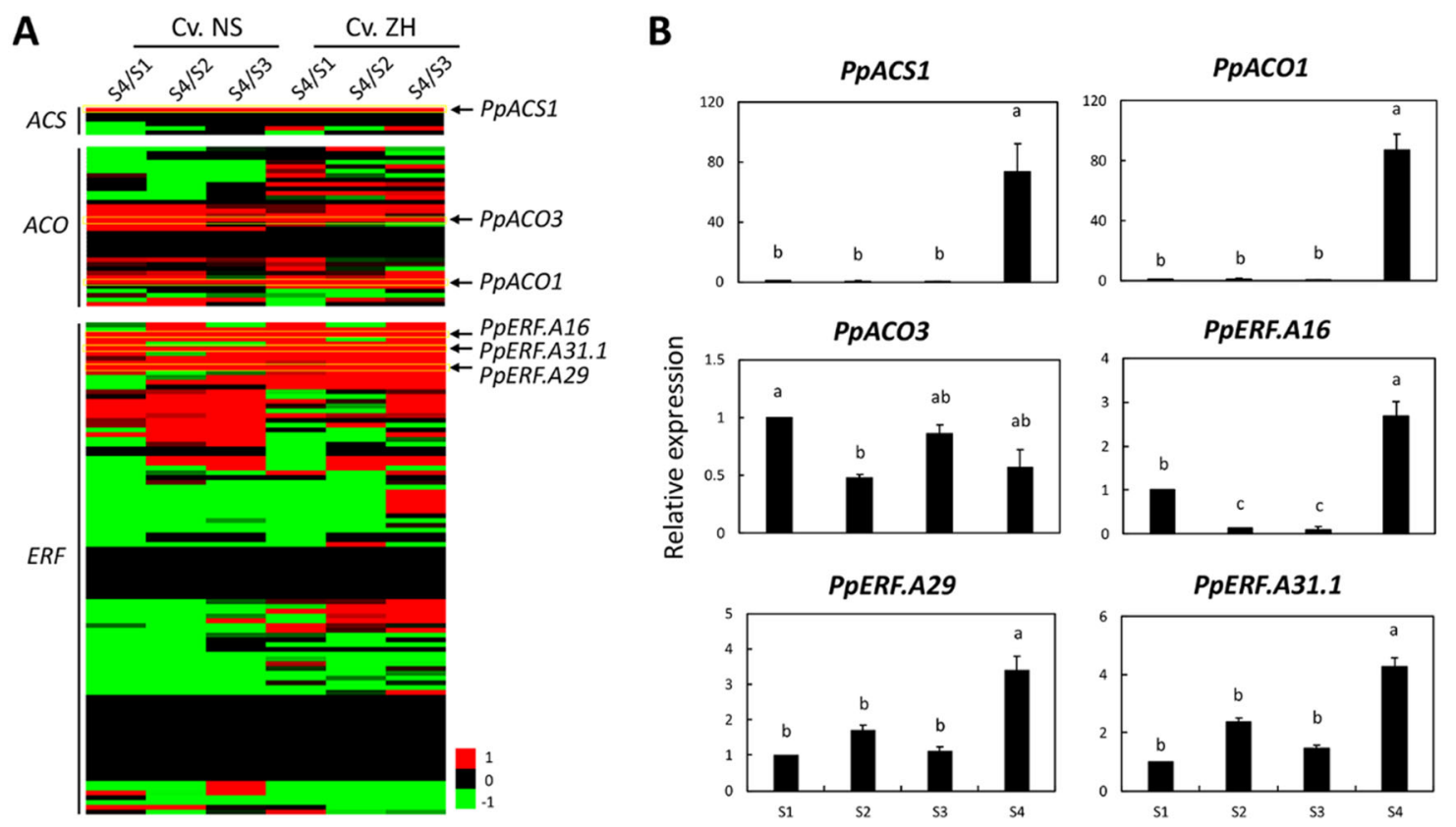

C
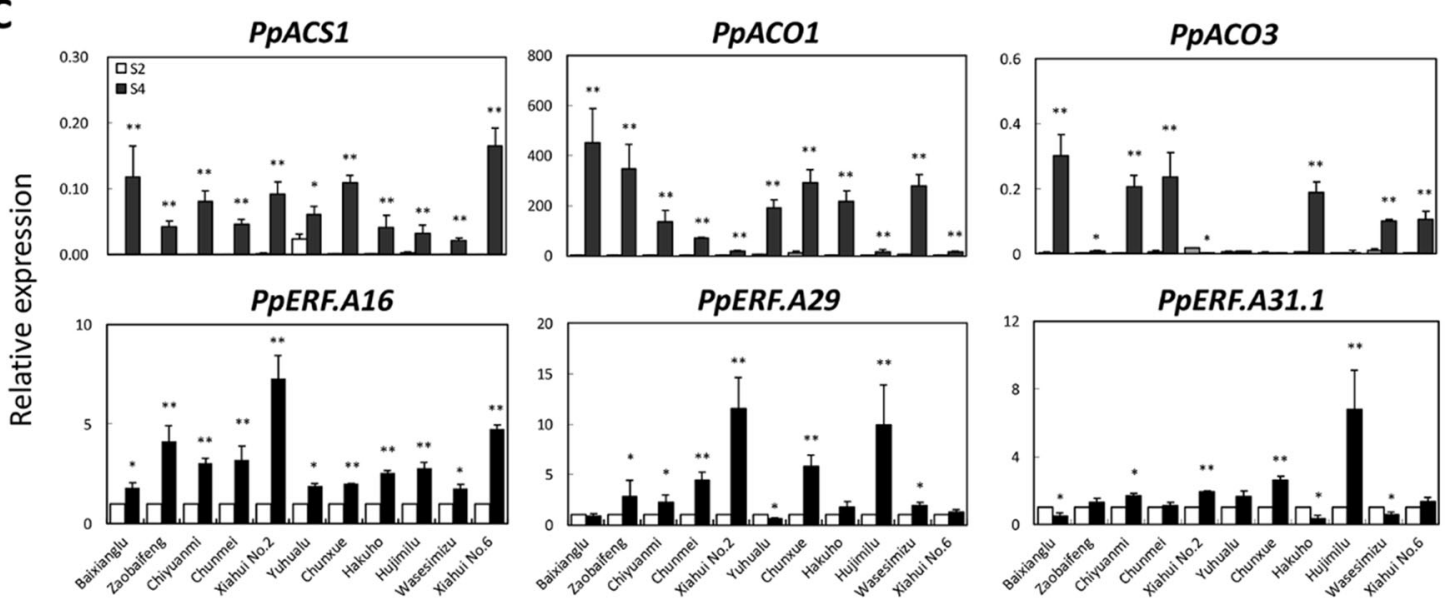

Fig. 1 Transcriptome-based identification of the $A C S, A C O$, and $A P 2 / E R F$ genes potentially associated with peach fruit ripening. A Transcriptome-based analysis of the differentially expressed ACS, ACO, and AP2/ERF genes between developing (S1 to S3) and ripening (S4) fruits in two peach cvs. NS and ZH. The color bar is calculated by $\log _{2}$ fold-change value of the ratio. B The expression profiles of PpACS1, PpACO1, PpACO3, PpERF.A16, PpERF.A29, and PpERF.A31.1 were determined by quantitative real-time PCR (qRT-PCR) in cv. Xiahui No.5 fruits at S1, S2, S3, and S4. C The expression levels of PpACS1, PpACO1, PpACO3, PpERF.A16, PpERF.A29, and PpERF.A31.1 were determined in the fruits of 11 peach cultivars at S2 and S4

Subsequently, yeast one-hybrid $(\mathrm{Y} 1 \mathrm{H})$ assays showed that PpERF.A16 binds to the PpACS1, PpACO1, and PpendoPGM promoters (Fig. 2C). Moreover, a PpERF.A16::HIS fusion protein was generated by inserting the whole sequences of PpERF.A16 into the pCold-TF vector. Kinetic assay showed that binding values between PpERF. A16::HIS, and the promoters of the three ripening-related genes gradually increased with increasing concentrations of PpERF.A16::HIS (Fig. 2D). Furthermore, electrophoretic mobility shift assay (EMSA) showed that PpERF. A16::HIS could bind to the probes of the PpACS1,
PpACO1, and PpendoPGM promoters, and the binding signals weakened with increasing concentrations of the cold probe (Fig. 2D). Taken together, PpERF.A16 directly binds to the PpACS1, PpACO1, and PpendoPGM promoters to enhance their activities.

\section{Effect of PpERF.A16 on ethylene biosynthesis}

To unravel the role of PpERF.A16 in ethylene biosynthesis, overexpression and antisense vectors of $P p E R F$. $A 16$ were individually constructed and then introduced to Agrobacterium tumefaciens. After introducing the 


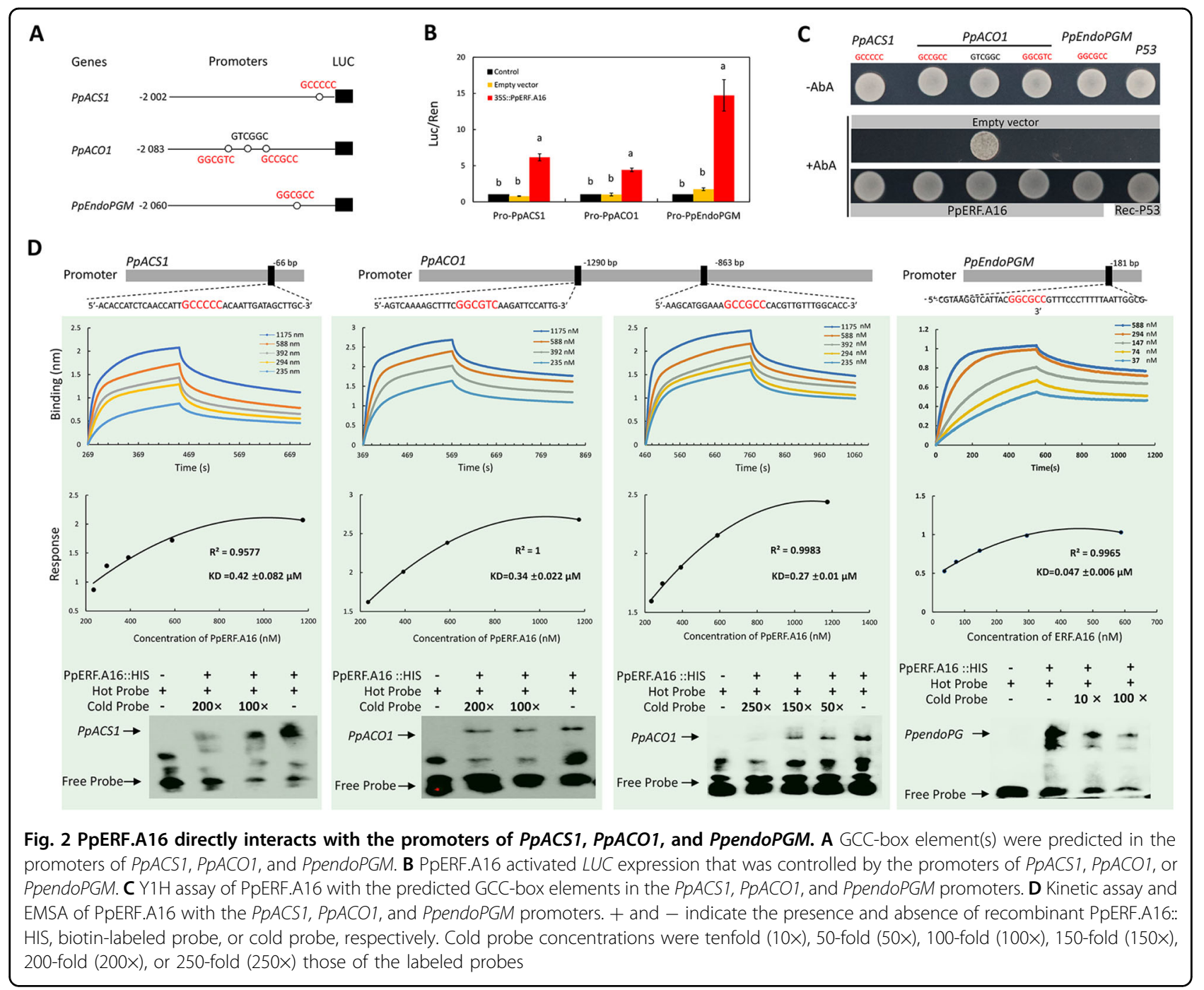

overexpression vector of PpERF.A16 into tobacco and identifying the positive lines (Fig. S4), the ethylene production rate was measured using a gas chromatography system. The results showed that the ethylene production rate was higher in the transgenic lines of PpERF.A16 $(P$ value $<0.001)$ than in the nontransgenic regeneration lines (Fig. 3A), suggesting that PpERF.A16 can promote ethylene biosynthesis in transgenic tobacco. Furthermore, Agrobacterium tumefaciens containing overexpression and antisense vectors of PpERF.A16 was independently injected into the fruits harvested at $\sim 10$ days before the commercial harvesting date, and ethylene production rates were measured at 3,4 , and 5 days after injection (DAI). At 5 DAI, the ethylene production rate was increased in the fruits overexpressing PpERF.A16 but decreased in the fruits silencing PpERF.A16 compared to the fruits infiltrated with the empty vector (Fig. 3B). Thus, PpERF.A16 can positively mediate ethylene biosynthesis during peach fruit ripening.
To test whether the expression of the PpACS1, PpACO1, and PpendoPGM genes was affected by PpERF. A16, qRT-PCR analyses were conducted on the transiently transformed peach fruits. As a result, both PpACS1 and $P p A C O 1$ were upregulated in fruits overexpressing PpERF.A16 but downregulated in the fruits silencing PpERF.A16 compared to the fruits infiltrated with the empty vector (Fig. 3C). Further qRT-PCR analysis showed that the transcript levels of PpERFA15.1/15.2/15.3 and PpERFA17.2/17.2 were most closely related to PpERF.A16 and were not downregulated in the fruits with silenced PpERF.A16 (Fig. S5). These results suggest that PpERF. A16 can positively regulate ethylene biosynthesis by inducing the expression of both PpACS1 and PpACO1. In addition, PpERF.A16 upregulated the expression of PpendoPGM (Fig. 3C) and ripening-related genes (such as PpCHI, PpPDS, PpZEP, and PpNECD) (Fig. S6). Together with the results of the promoter analyses described above, PpERF.A16 was shown to be able to 


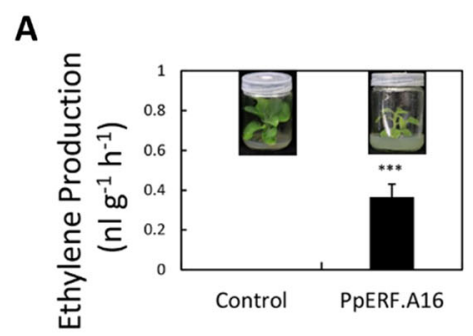

B
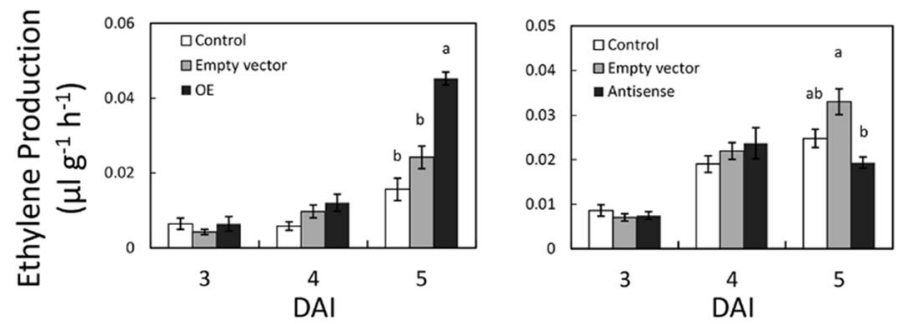

C
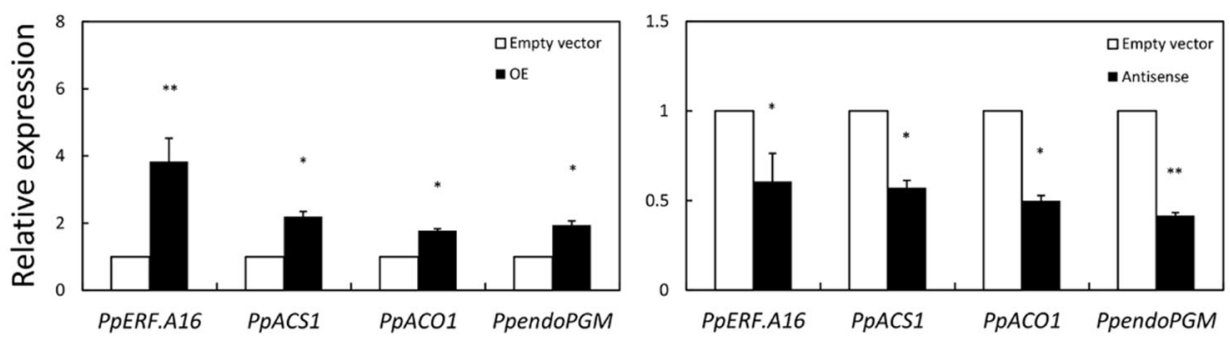

Fig. 3 PpERF.A16 positively mediates ethylene biosynthesis. A The ethylene production rate was increased in tobacco transgenic plants overexpressing PDERF.A16 compared to the wild-type control. B The peach fruits harvested $\sim 10$ days before the commercial harvesting date were transiently transformed with an empty vector or a vector overexpressing PpERF.A16 (OE) or antisense PpERF.A16 (Antisense). Ethylene production rates were measured at 3,4, and 5 days after injection (DAl) of Agrobacteria containing the vectors and showed a significant increase in the OE fruits but a decrease in the antisense fruits compared to the fruit injected with the PCAMBIA1301 empty vector and noninjected control fruit at 5 DAI. C The expression levels of PpERF.A16, PpACS1, PpACO1, and PpendoPGM genes were increased in the OE fruits but decreased in the antisense fruits compared to the empty vector fruits at 5 DAl

directly affect the expression of the PpACS1, PpACO1, and PpendoPGM genes.

\section{Identification of the NAC TF coexpressed with PpERF.A16}

To mine the upstream TF(s) of PpERF.A16 expression and ethylene biosynthesis, the potential cis-regulatory elements in the PpERF.A16 promoter were predicted, revealing 12 elements (the sequences with red color) for the NAC TF (Fig. S7). A higher number of elements for the NAC TF indicated that this TF should play an important role in mediating PpERF.A16 expression and ethylene production. Subsequently, 103 members of the $N A C$ gene family were isolated from the peach genome. Together with the NAC genes derived from other fruit trees, the $N A C$ gene family can be divided into two groups, A and B (Fig. S8 and Table S3). Of the two groups, group A comprised 69 clusters (A1 $\rightarrow$ A69), while group B contained only three clusters (B1 $\rightarrow$ B3; Fig. S8). Based on the RNA-Seq data of fruit samples of cvs. NS and ZH in a previous study ${ }^{34}$, two NAC genes were more highly expressed in ripening fruit than in developing fruit of both cvs. NS and ZH (Fig. 4A and Table S2). These two genes, Ppa007445m and Ppa008828m, belong to clusters A32 and A59 and were thus designated PpNAC.A32 and PpNAC.A59, respectively (Table S3). Of the two genes, however, PpNAC.A32 had similar levels of expression between developing and ripening fruits of cv. Xiahui No.5
(Fig. 4B) and cvs. Xiahui No. 2, Yuhualu, Chunxue, and Wasesimizu (Fig. 4C), while PpNAC.A59 was more highly expressed in ripening fruit than in developing fruit of all 12 tested cultivars (Fig. 4B, C). Obviously, PpNAC.A59 had an identical expression profile to PpERF.A16 in all tested peach cultivars, indicating that this TF may induce the expression of PpERF.A16 and is probably associated with peach fruit ripening.

\section{Interaction of PpNAC.A59 with the PpERF.A16 promoter}

To test whether PpNAC.A59 interacts with the PpERF. $A 16$ promoter, a dual-luciferase assay was conducted and showed an interesting result. The $L U C$ gene driven by the PpERF.A16 promoter had higher activity in Arabidopsis protoplasts overexpressing PpNAC.A59 (35S::PpNAC. A59) than in Arabidopsis protoplasts transformed with an empty vector or without any transformation (control; Fig. $5 \mathrm{~A}$ ). In contrast, the $L U C$ gene driven by any promoter of PpACS1, PpACO1, and PpendoPGM had similar activity in Arabidopsis protoplasts overexpressing PpNAC.A59 to that in Arabidopsis protoplasts transformed with an empty vector (Fig. 5A). Therefore, PpNAC.A59 interacts with the PpERF.A16 promoter.

To determine which fragment(s) in the promoter of PpERF.A16 could be bound by PpNAC.A59, the promoter sequences of $P p E R F . A 16$ were fragmented into seven sections $(\mathrm{I} \rightarrow \mathrm{VII})$. Only fragment V was bound by PpNAC. 
A

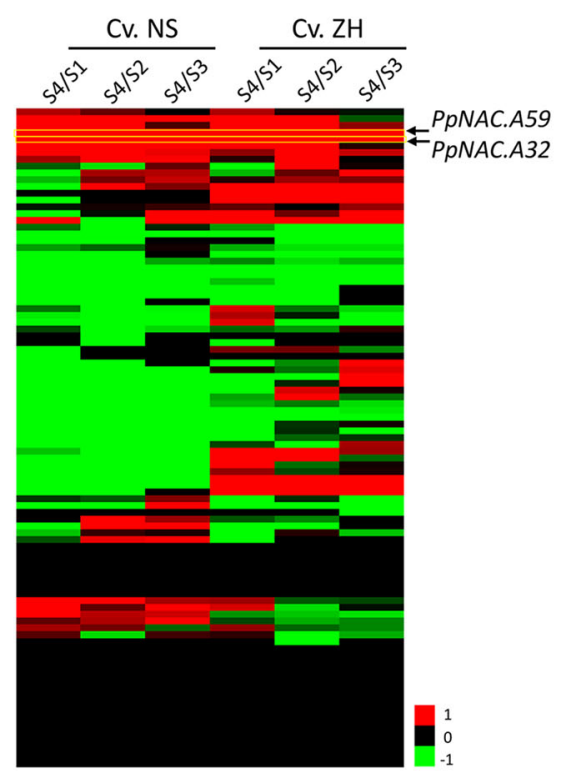

C

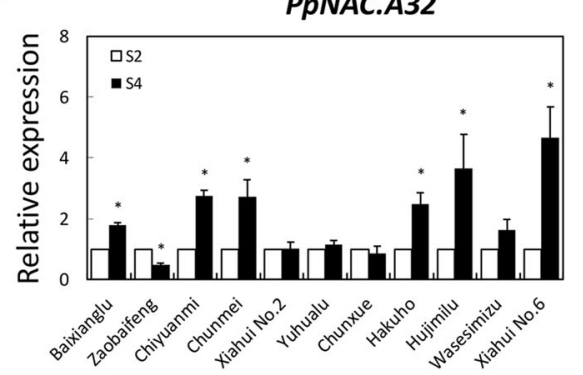

B

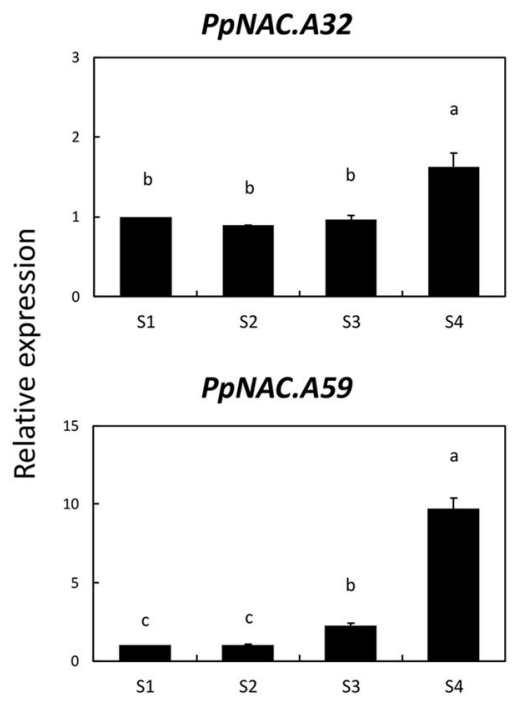

PpNAC.A59

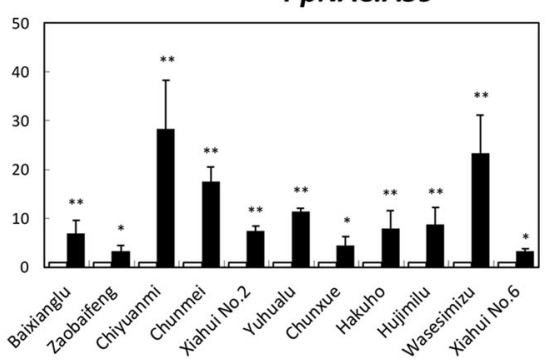

Fig. 4 Identification of the NAC genes probably associated with peach fruit ripening. A Transcriptome analysis showed that PpNAC.A59 and PpNAC.A32 were differentially expressed between developing (S1 to S3) and ripening (S4) fruits in peach cvs. NS and ZH. The color bar was calculated by the $\log _{2}$ fold-change values of the ratio. B The expression profiles of PpNAC.A59 and PpNAC.A32 were tested by qRT-PCR in cv. Xiahui No. 5 fruits at S1, S2, S3, and S4. C The expression levels of PpNAC.A59 and PpNAC.A32 were tested in the fruits of 11 peach cultivars at S2 and S4

A59 in yeast cells (Fig. 5B). In fragment V, we detected two possible cis-regulatory elements (WNNYBTNNNNNN NAMGNHW and TTRCGT) for NAC TF ${ }^{42-44}$, and these elements were used to synthesize the biotin-labeled probes (Fig. 5C). Subsequently, a recombinant PpNAC.A59::HIS fusion protein was generated by inserting the whole sequences of PpNAC.A59 into the pCold-TF vector. Kinetic assay showed that the binding value between PpNAC.A59::HIS and each of the three probes gradually increased with increasing concentrations of PpNAC.A59:: HIS, and the binding response between PpNAC.A59::HIS and the probe boxed with a yellow color peaked when the concentration reached $4500 \mathrm{nM}$ (KD $<1 \mu \mathrm{M}$; Fig. 5C). Moreover, EMSA showed that PpNAC.A59::HIS could bind to the probe boxed with a yellow color, and the binding signal weakened as the concentration of the cold probe increased (Fig. 5C). Taken together, PpNAC.A59 can directly bind to the promoter of PpERF.A16.

\section{Effect of PpNAC.A59 on ethylene biosynthesis}

To unravel the role of PpNAC.A59 in ethylene biosynthesis, overexpression and antisense vectors of PpNAC.A59 were constructed and then introduced into A. tumefaciens. The resuspended Agrobacterium was injected into peach fruits, and ethylene production rates were measured at 3, 4, and 5 DAI. At 5 DAI, the ethylene production rate was increased in the fruits overexpressing PpNAC.A59 but reduced in the fruits with silenced PpNAC.A59 compared to the fruits injected with an empty vector (Fig. 6A). This result indicated that PpNAC. A59 can positively regulate ethylene biosynthesis during peach fruit ripening.

To test whether the expression of ethylene biosynthesis and ripening-related genes was affected by PpNAC.A59, the transiently transformed peach fruits were analyzed using qRT-PCR. The results showed that the expression levels of PpACS1, PpACO1, and PpERF.A16 were 


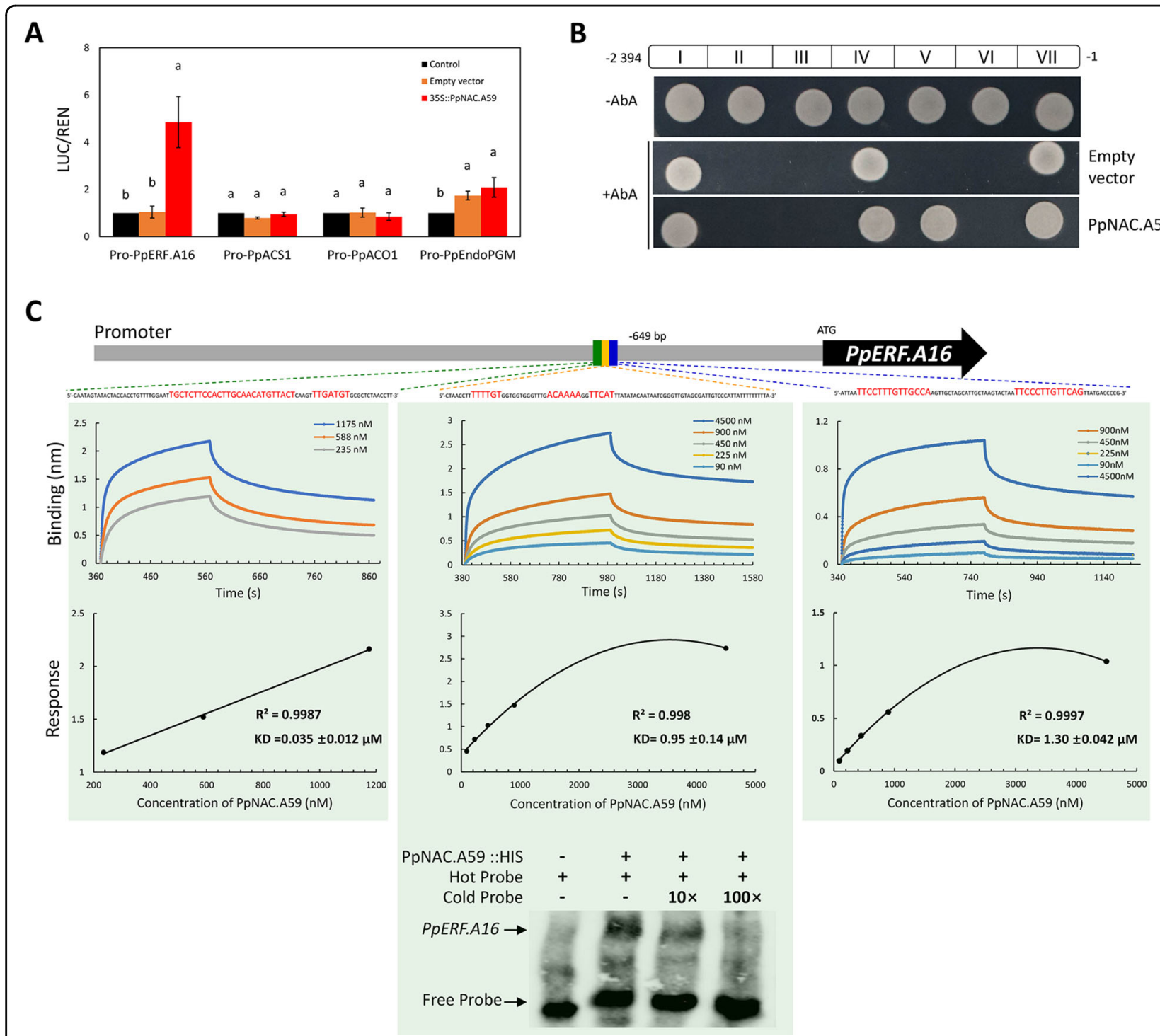

Fig. 5 PpNAC.A59 directly interacts with the PpERF.A16 promoter. A PpNAC.A59-activated LUC expression, which was controlled by PpERF.A16 promoter. B Y1H assay of PpNAC.A59 with seven fragments (I to VII) in the PpERF.A16 promoter. C Kinetic assay and EMSA of PpNAC.A59 with the PPERF.A16 promoter. "+" and "-" indicate the presence and absence of recombinant PpNAC.A59::HIS, biotin-labeled probe, or cold probe, respectively. Cold probe concentrations were tenfold (10x) and 100-fold (100x) those of the labeled probes

upregulated in fruits overexpressing PpNAC.A59 and downregulated in the fruits with silenced PpNAC.A59 compared to the fruits infiltrated with the empty vector (Fig. 6B). Further qRT-PCR analysis showed that the transcript levels of PpNAC.A58/A50/A61, which were most closely related to PpNAC.A59, were not downregulated in the fruits with silenced PpNAC.A59 (Fig. S9). In addition, PpNAC.A59 upregulated the expression of PpendoPGM (Fig. 6B) and other ripening-related genes (such as $P p C H I, P p Z E P$, and $P p N E C D$ ) (Fig. S10). Due to no interaction being detected between PpNAC.A59 and the promoter sequences of PpACS1, PpACO1, and PpendoPGM (Fig. 5A), it is likely that PpNAC.A59 indirectly promotes the transcription of these genes via the PpERF.A16-ethylene pathway.

\section{Discussion}

PpERF.A16 directly regulates ethylene biosynthesis during fruit ripening

AP2/ERF TFs have been characterized in plant species, including peach ${ }^{45,46}$, and are involved in various biological processes, such as plant growth, development, senescence, and stress tolerance ${ }^{6,47}$. In fleshy fruits, AP2/ERF TFs play important roles in transmitting the ethylene signal and stimulating activities of downstream genes responsive to fruit ripening ${ }^{6,19}$. This type of $A P 2 / E R F$ gene has been 

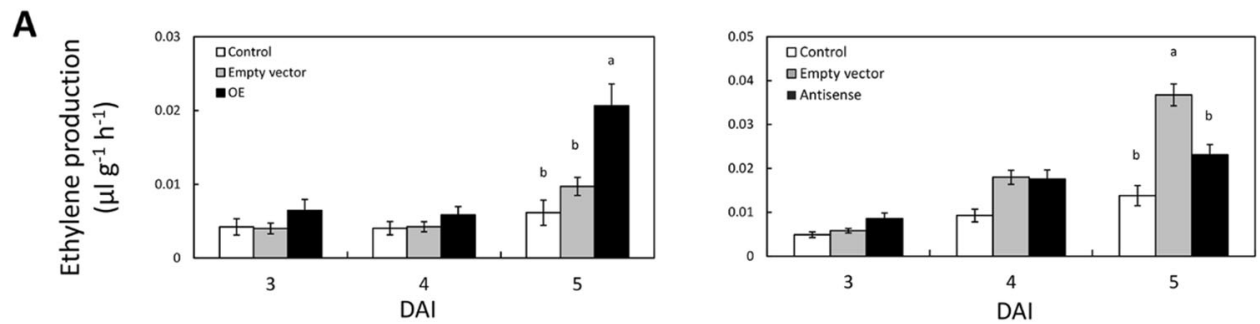

B
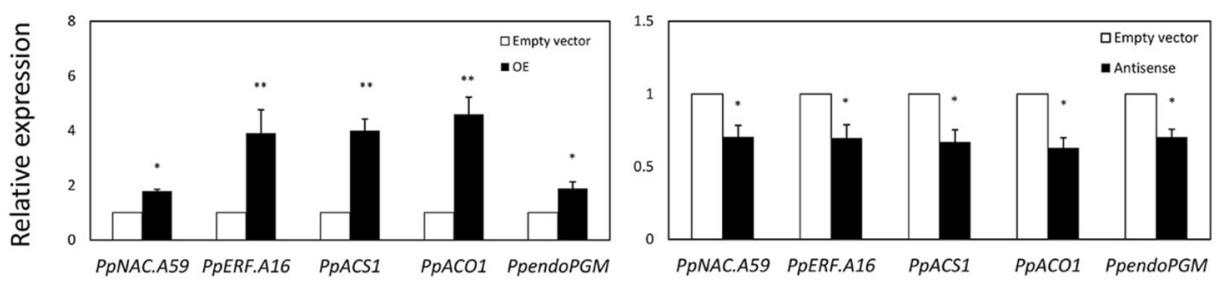

Fig. 6 PpNAC.A59 positively mediates ethylene biosynthesis. A The peach fruits harvested $\sim 10$ days before the commercial harvesting date were transiently transformed with an empty vector or a vector overexpressing PpNAC.A59 (OE) or antisense PpNAC.A59 (Antisense). Ethylene production rates were measured at 3,4, and 5 days after injection (DAl) of Agrobacteria containing the vectors and showed a significant increase in the OE fruits but a decrease in the antisense fruits compared to the fruits injected with the empty vector PCAMBIA1301 and noninjected control fruits at 5 DAI. B The expression levels of PpERF.A16, PpACS1, PpACO1, and PpendoPGM genes were increased in the OE fruits but decreased in the antisense fruits compared to the empty vector fruits at 5 DAI

identified in climacteric fruits such as apple, banana, and pear $^{11-13}$. This study identified a similar gene PpERF.A16 from previously reported RNA-Seq data from peach fruit. Overexpression of PPERF.A16 could enhance the expression of PpACS1 and PpACO1 and thus increase ethylene production during peach fruit ripening (Fig. 3). Furthermore, PpERF.A16 could also induce the expression of other ripening-related genes (Figs. 3C, S6) and is thus likely associated with peach fruit ripening. PpERF. $A 16$ showed a similar function to the previously reported ERF genes MdERF3, PbERF24, and MaERF9 involved in regulating the ripening of climacteric fruits ${ }^{11-13}$. However, a difference is that PpERF.A16 binds to the promoters of both the PpACS1 and PpACO1 genes (Fig. 2), while MdERF3, PbERF24, and MaERF9 interact with the promoters of an ACS or ACO gene but not both genes $^{11-13}$. Another difference is that these genes are clustered into different subgroups of the ERF family, i.e., PpERF.A16, PbERF24, MdERF3, and MaERF9 were clustered into the A16, A17, A39 and A13 subgroups, respectively (Table $\mathrm{S} 1$ ).

It has been reported that exogenous auxin can effectively induce the expression of PpACS1 and PpERF. $A 16^{37,48}$. Ethylene production is associated with the TC microsatellite genotypes of $P p Y U C 11$ in peach fruits with flesh melting and stony hard phenotypes ${ }^{49,50}$. Recently, it was confirmed that auxin can promote ethylene biosynthesis in apple fruit by activating MdARF ${ }^{16}$. MdARF 5 can bind to the MdACS1, MdACS3a, MdACO1, and $M d E R F 2$ promoters to induce their expression in apple fruit. It is possible that auxin induces ethylene biosynthesis in peach fruit by an ARF TF. However, exogenous auxin treatment cannot upregulate ARF TFs in peach fruit $^{37,48}$. Instead, the expression levels of auxin/ indole-3-acetic acid (auxin/IAA) genes (IAA11, IAA13, and IAA9_2) were increased in the peach fruits with exogenous auxin treatment compared with those in the control fruits ${ }^{37,48}$. It is more possible that these auxin/IAA TFs directly or indirectly induce the expression of PpACS1 and PpERF.A16 to promote ethylene biosynthesis in peach fruit.

\section{PpNAC.A59 indirectly mediates ethylene biosynthesis during fruit ripening}

Plant-specific NAC TFs of woody and grassy plants have been phylogenetically analyzed ${ }^{51,52}$ and are reported to regulate various physiological traits ${ }^{53-56}$. NOR was the first $N A C$ gene known to promote ethylene biosynthesis in a nonripening tomato mutant ${ }^{22}$. Subsequently, the SINAC4 and NOR-like 1 genes were proven to be associated with fruit ripening in tomato ${ }^{21,23}$, similar to $A d N A C 6$ and $A d N A C 7$ in kiwifruit ${ }^{25}$. In the present study, PpNAC.A59 was shown to have a higher level of expression in ripening fruit than in developing fruit of all tested peach cultivars (Fig. 4). Interestingly, PpNAC.A59 cannot interact with the promoters of $P p A C S 1$ and $P p A C O 1$ (Fig. 5A) but could induce the expression of both $P p A C S 1$ and PpACO1 to promote ethylene biosynthesis in transiently transformed fruits (Fig. 6). This result is different from previous studies of the NACs associated with ethylene biosynthesis during fruit ripening. For example, NOR-like 1 of tomato interacts with the SlACS2 and 
SlACS4 promoters $^{21}$, and AdNAC6/7 of kiwifruit interacts with the AdACS1 and $A d A C O 1$ promoters ${ }^{25}$. In contrast, PpNAC.A59 directly interacts with the PpERF.A16 promoter (Fig. 5) but not with the PpACS1 or PpACO1 promoter. Then, PpERF.A16 interacts with the promoters of PpACS1 and PpACO1. Obviously, PpNAC.A59 indirectly mediates ethylene biosynthesis during peach fruit ripening. It is noteworthy that PpNAC.A59 was not clustered together with any of the previously reported NACs associated with ethylene biosynthesis during fruit ripening. PpNAC.A59, AdNAC6/7, NOR/NOR-like 1, SINAC1, and SINAC4 were individually clustered into subgroups A59, A21, A35, A25, and A24 of the NAC family, respectively (Table S3).

In previous studies, NACs were reported to be involved in abscisic acid (ABA) biosynthesis ${ }^{57,58}$. Both Oryza sativa OsNAC2 and Solanum lycopersicum SINAP2 directly control the expression of the ABA biosynthesis gene 9-cis-epoxycarotenoid dioxygenase and the ABA catabolic gene $A B A$ 8'-hydroxylase to affect $A B A$ production ${ }^{59,60}$. It is noteworthy that $A B A$ indirectly promotes fruit ripening by triggering ethylene biosynthesis in climacteric fruits ${ }^{61,62}$. In this study, we found that PpNAC.A59 positively regulated the expression of $\mathrm{ABA}$ biosynthesis genes (Zeaxanthin epoxidase and 9-cis-epoxycarotenoid dioxygenase; Fig. S10), indicating that PpNAC.A59 is likely involved in ABA biosynthesis in peach fruit. Therefore, ethylene biosynthesis may also be influenced by the PpNAC.A59-ABA-ethylene signal cascade during peach fruit ripening.

\section{Molecular network of fruit ripening in peach}

Controlling the ripening process of climacteric fruits is important to the commercialization of these fruit crops and has been widely studied at the molecular genetics level ${ }^{19,63}$. In tomato, a genetic network regulating fruit ripening has been established and consists of TFs of different families, including RIN, FUL1, FUL2, HB-1, AP2a, ERF6, TAGL1, NOR, CNR, and TDR4 ${ }^{29,30}$. This network was further enriched by the most recently identified TFs, SINAC4, SINAC1, SIZFP2, and NOR-like $1^{20,21,23,24}$, and epigenetic modifiers, such as DNA methylation $^{64,65}$, microRNA ${ }^{66,67}$, and long noncoding RNA $^{68,69}$. Obviously, the understanding of the genetic network regulating fruit ripening in tomato is much advanced compared to our understanding of the network in tree fruits. During apple fruit ripening, the regulatory network of ethylene biosynthesis was constructed to include the TFs MYC2, ARF5, ERF2, and ERF3 and ethylene biosynthesis genes ${ }^{12,15,16}$. However, in other perennial fruit trees, few TFs were concatenated into a network. In peach fruit, two TFs, PpHB.G7 and PpTCP.A2, were identified in the previous studies ${ }^{40,41}$. Of these two TFs, the transcription activator PpHB.G7 can directly bind to the $P p A C S 1$ and $P p A C O 1$ promoters to increase ethylene production ${ }^{40}$, while the transcription repressor PpTCP.A2 can inhibit the expression of PpACS1 to hinder ethylene biosynthesis ${ }^{41}$.

In the present study, two TFs, PpERF.A16 and PpNAC.A59, were shown to be involved in ethylene biosynthesis during peach fruit ripening. These results could be integrated into a primary network of fruit ripening in peach (Fig. 7). First, PpNAC.A59 can induce the expression of PpERF.A16 by directly binding to its promoter. Second, PpERF.A16 and PpHB.G7 enhances the expression of both PpACS1 and PpACO1 by directly interacting with their promoters, contributing to an increase in ethylene production. Finally, ethylene accelerates fruit ripening by mediating fruit metabolism, such as flavonoid and carotenoid metabolism. It is noteworthy that this conclusion is supported by a previous study in which the NAC TF was predicted to control ethylene biosynthesis during peach fruit ripening $^{70}$. The only difference is that the predicted NAC TF can induce the expression of ethylene biosynthesis genes by directly interacting with their promoters ${ }^{70}$, while PpNAC.A59 indirectly induces the expression of both PpACS1 and PpACO1 by directly mediating the expression of PpERF.A16. Further work will focus on the enrichment of this network by identifying more TFs associated with fruit ripening.

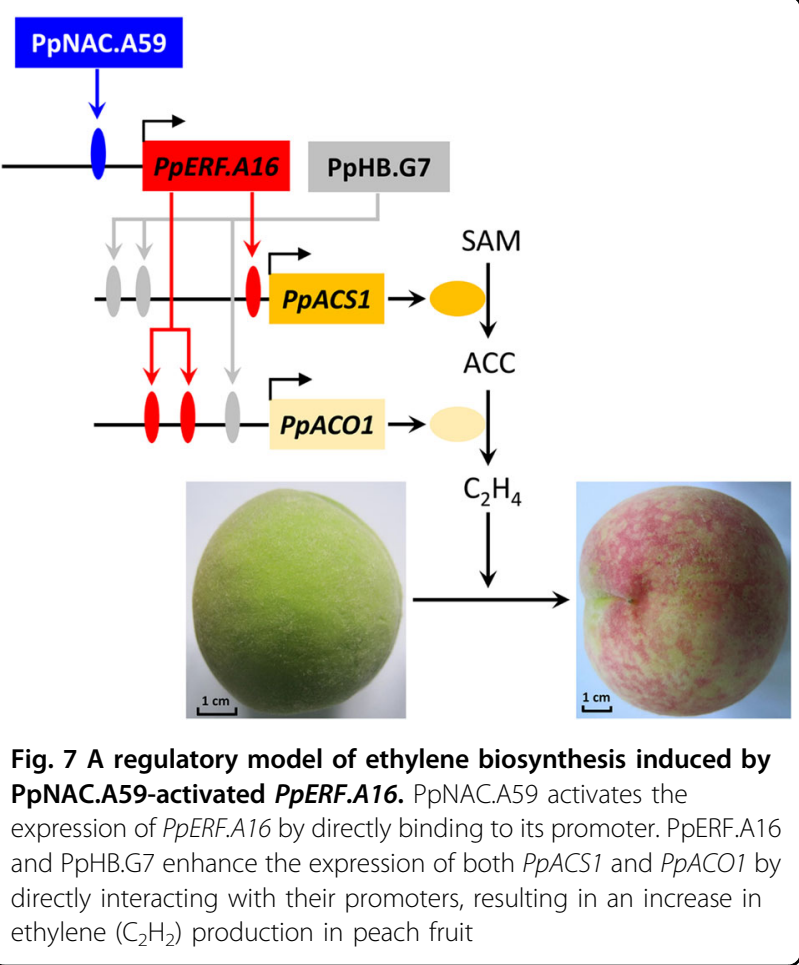




\section{Materials and methods}

\section{Plant materials}

A total of 12 peach cultivars were used in this study. Of these cultivars, Wasesimizu and Xiahui No. 5 were maintained at the Jiangsu Academy of Agricultural Sciences (Nanjing, Jiangsu Province, China). The other ten cultivars (Chunxue, Hakuho, Hujinmilu, Xiahui No. 2, Yuhualu, Chiyuanmi, Chunmei, Xiahui No. 6, Baixianglu, and Zaobaifeng) were identical to those in a previous study ${ }^{40}$. The cv. Baixianglu fruit presents a semifreestone melting flesh phenotype, and the fruits of the other ten peach cultivars present a clingstone melting flesh phenotype. Based on the dynamic investigation of fruit development and ripening ${ }^{71}$, the fruits of cv. Xiahui No. 5 were collected at the fruitlet (S1), stone hardening (S2), preripening (S3), and ripening (S4) stages, and the fruits of the other 11 cultivars were collected at the rapid enlargement (roughly corresponding to S2) and ripening stages (S4; Table S4). Grouping, biological replication, and storage of fruit samples were as described in a previous study ${ }^{40}$.

\section{Sequence and phylogenetic analyses of the ACS, ACO, AP2/} $E R F$, and $N A C$ genes

To isolate the $A C S, A C O, A P 2 / E R F$, and $N A C$ genes in peach and other fruit trees, the nucleotide and amino acid sequences of the $A C S, A C O, A P 2 / E R F$, and $N A C$ genes in peach were extracted from the peach genome version 1.0 (Genome Database for Rosaceae; http://www. rosaceae.org/). Then, these downloaded sequences were used as indexes to find their homologs in papaya (Carica papaya ASGPBv0.4), strawberry (Fragaria vesca v 1.1), orange (Citrus sinensis v1.1), grape (Vitis vinifera Genoscope.12X), apple (Malus domestica v1.0) (https:// phytozome.jgi.doe.gov/), and pear (Pyrus bretschneideri; http://peargenome.njau.edu.cn/) genomes (Tables S1, S3). Sequence alignment and phylogenetic analyses were as described in a previous study ${ }^{40}$.

\section{Gene expression analysis in developing and ripening fruits}

In a previous study, RNA-Seq was performed in the developing and ripening fruits of three peach cultivars, Nanshantiantao (NS), Zhaohui $(\mathrm{ZH})$, and Myojo ${ }^{34}$. Of these three cultivars, NS and $\mathrm{ZH}$ fruits presented a melting flesh phenotype, and the fruit hardness gradually decreased during ripening, while fruits of the Myojo cultivar did not soften during fruit ripening. For this reason, the transcriptome data fruits from cvs. NS and $\mathrm{ZH}$ were used to isolate the $A C S, A C O, A P 2 / E R F$, and $N A C$ genes that may be associated with fruit ripening. The hierarchical clustering of transcriptome data was performed according to a previous study ${ }^{40}$. By comparing the gene expression levels of the ripening fruit (S4) to those of the developing fruits ( $\mathrm{S} 1$ to $\mathrm{S} 3$ ), we isolated the target genes PpACS1, PpACO1, PpACO3, PpERF.A16, PpERF.A29,
PpERF.A31.1, PpNAC.A32, and PpNAC.A59. Thus, these eight genes were further tested by qRT-PCR analysis. The details for qRT-PCR and data analysis were identical to those of a previous study ${ }^{40}$.

\section{Transformations in peach fruit and tobacco}

The full length CDSs of PpERF.A16 and PpNAC.A59 were amplified with the PCR primers listed in Table S5 and inserted in a sense orientation downstream of the CaMV 35S promoter in the pCAMBIA1301 vector to generate gene overexpression vectors. An approximately 400-bp fragment of the CDS of PpERF.A16 or PpNAC. $A 59$ was amplified using the primers listed in Table S5 and inserted in an antisense orientation downstream of the CaMV $35 \mathrm{~S}$ promoter in the pSAK277 vector to create a gene silencing construct. The $400 \mathrm{bp}$ fragments were located in the nonconserved region and showed no significant similarity (defined as no $>21$ bp in length with $100 \%$ identity) to the sequences of the most closely related genes within the same gene family (Figs. S11-S20). pCAMBIA1301 contains a hygromycin resistance gene, and pSAK277 contains a kanamycin resistance gene as a selection marker in plant transformation. These vectors were transferred into Agrobacterium tumefaciens EHA 105 cells that were used to transiently transform peach fruit tissues with a previously reported Agrobacterium infiltration protocol ${ }^{40}$. The fruit used for the transformation experiments was harvested from the peach cultivar Xiahui No. 5 at $\sim 10$ days before the commercial harvesting date. At three, four and five DAI, at least five fruits were sealed in a 5 - $\mathrm{L}$ airtight jar, and six jars were used for each construct. Three DAI, ethylene production was measured every day. Fruit flesh tissues were sampled at 5 DAI for qRT-PCR analysis. All of the PCR primer sequences are listed in Supplementary Table S5.

Overexpression vectors of PpERF.A16 were also introduced into tobacco (Nicotiana tabacum) based on Agrobacterium-mediated transformation as described previously $^{72}$. The regenerated tobacco plants were tested by PCR for amplification of the hygromycin gene from the pCAMBIA1301 vector. PCR-positive plants and wild-type control plants were used for measuring ethylene production.

\section{Ethylene measurements}

The ethylene production rate was measured using a gas chromatography system (GC2010, Shimadzu, Japan) with a $1000 \mu \mathrm{l}$ rheodyne injector (Gaoge, Shangshai, China) according to a previously reported procotol ${ }^{73}$ with a few modifications. In brief, at room temperature, at least four fruits were weighed as a replicate and then placed into a 3-1 airtight chamber. For tobacco, each plant was a replicate and individually weighed after collection from tissue culture medium and placed into a $15 \mathrm{ml}$ centrifuge 
tube. Six replicates were used for each gene construct and each control. A $0.5-\mathrm{ml}$ gas sample was collected from the chambers and tubes and injected into a gas chromatograph fitted with a $30-\mathrm{cm}$ glass column (3.2 $\mathrm{mm} \mathrm{ID).} \mathrm{Gas}$ flows for $\mathrm{N} 2, \mathrm{H} 2$, and air were 40,40 , and $500 \mathrm{ml} / \mathrm{min}$, respectively. The oven, injector, and FID temperatures were 300,60 , and $260^{\circ} \mathrm{C}$, respectively.

\section{Protoplast isolation and dual-luciferase assay}

Protoplast isolation was as described in a previous report ${ }^{40}$. Approximately 2000-bp sequences upstream of the initiation codons of PpACS1, PpACO1, PpendoPGM, and PpERF.A16 were inserted into a pGreenII 0800-LUC vector for a dual-luciferase assay that was performed according to a previous study ${ }^{40}$. At least six biological replicates were used in each assay. All of the primer sequences are listed in Table S5.

\section{Yeast one-hybrid assay $(\mathrm{Y} 1 \mathrm{H})$}

It is well known that ERF TFs can bind to the GCC-box element $^{74,75}$. Thus, the possible GCC-box elements were surveyed from the promoters of PpACS1, PpACO1, and PpEndoPGM (Fig. 2A). The fragments containing the possible GCC-box element were amplified from the genomic DNA of cv. Xiahui No.5 and then inserted into the pAbAi vector. The whole sequences of PpERF.A16 and PPNAC.A59 genes were individually inserted into the pGADT7 vector. The $\mathrm{Y} 1 \mathrm{H}$ assay was performed using the Matchmaker Gold Yeast One-Hybrid Library Screening System (Clontech, Palo Alto, CA). All of the primer sequences are listed in Table S5.

\section{Recombinant proteins of both PpERF.A16 and PpNAC.A59 in Escherichia coli}

The whole sequences of PpERF.A16 and PpNAC.A59 were individually inserted into the pCold-TF expression vector (including a HIS tag) to create recombinant proteins. Recombinant plasmids were introduced into Escherichia coli cells and incubated at $37^{\circ} \mathrm{C}$ until an $\mathrm{OD}_{660}$ of 0.6 was reached. Protein expression was induced at $16^{\circ} \mathrm{C}$ for $24 \mathrm{~h}$, and the recombinant protein was extracted and purified at $4{ }^{\circ} \mathrm{C}^{76}$. SDS-PAGE was conducted to analyze the extracted proteins. All of the primer sequences are listed in Table S5.

\section{Kinetic assay}

Fragments containing possible protein-binding elements were used to synthesize probes biotinylated at the ends of both the N- and C-termini (Sangon, Shanghai, China). Protein-DNA interactions were determined by biolayer interferometry using a ForteBio Octet 96 Red Instrument (Menlo Park, CA, USA). The kinetic assay comprised the following five steps: (1) baseline acquisition was conducted by loading PBS buffer $\left(1.4 \mathrm{mM} \mathrm{KH}_{2} \mathrm{PO}_{4}\right.$,
$2.7 \mathrm{mM} \mathrm{KCl}, 4.3 \mathrm{mM} \mathrm{Na} \mathrm{HPO}_{4}, 137 \mathrm{mM} \mathrm{NaCl}, 0.02 \%$ $(\mathrm{w} / \mathrm{v})$ Tween-20; $\mathrm{pH}=7.2)$ onto streptavidin-coated biosensors, (2) the biotin-labeled probe was loaded onto the sensor, (3) a second baseline acquisition was conducted with the same PBS buffer, and steps (4) and (5) were the respective association and dissociation between the recombinant protein and the biotin-labeled probe(s). Equilibrium binding affinities (KD) were measured using nanomole quantities of samples as well as rates of association and dissociation $(\mathrm{KA} / \mathrm{KD})^{77}$.

\section{Electrophoretic mobility shift assay (EMSA)}

The recombinant proteins and biotin-labeled probes were also used for EMSAs. In brief, the binding reaction was performed in a $0.2 \mathrm{ml}$ centrifuge tube for $20 \mathrm{~min}$ at room temperature, and the reaction mixtures contained $2 \mu \mathrm{l}$ loading buffer, $1 \mathrm{mg}$ poly $(\mathrm{dI} \cdot \mathrm{dC}), 5 \mathrm{mM} \mathrm{MgCl}_{2}, 2.5 \%$ glycerol, $0.05 \% \mathrm{NP}-40,200 \mathrm{nM}$ probe, and $1 \mu \mathrm{g}$ recombinant protein, with ultrapure water added for a total volume of $20 \mu \mathrm{l}$. For probe competition, 2, 10, 20, 30, 40, or $50 \mu \mathrm{M}$ nonlabeled probe was individually mixed into the reaction mixtures. After running in a $6 \%$ polyacrylamide gel, the protein-DNA complexes were electrophoretically transferred to a nylon membrane (GE Healthcare, Danderyd, Sweden). SDS-PAGE imaging was performed by a Tanon 5200 chemiluminescence imaging system (Tanon, Shanghai, China) and scanned using a ChemiDoc $^{\mathrm{TM}}$ XRS+ System (BioRad, Hercules, CA).

\section{Statistical analysis}

Means and standard errors were calculated from at least three biological replicates for qRT-PCR analysis, five biological replicates for ethylene measurement in tobacco, and at least six replicates for ethylene measurement in peach fruit. Analysis of variance was calculated by Student's $t$ test. The lowercase letters $\mathrm{a}, \mathrm{b}$, and $\mathrm{c}$ indicate the level of significance at $P<0.05$. Single, double and triple asterisks represent the levels of significance at $P<0.05$, $<0.01$, and $<0.001$, respectively.

\section{Acknowledgements}

We thank LetPub (www.letpub.com) for its linguistic assistance during the preparation of this paper. This work was supported by the Natural Science Foundations of China (31672118 and 31471856) and the Natural Science Foundations of Jiangsu Province (BK20140756).

\section{Author details}

${ }^{1}$ College of Horticulture/State Key Laboratory of Crop Genetics and Germplasm Enhancement, Nanjing Agricultural University, 210095 Nanjing, China. ${ }^{2} \mathrm{New}$ Zealand Institute of Plant \& Food Research Ltd, Private Bag 92169, Auckland 1142, New Zealand. ${ }^{3}$ Institute of Pomology, Jiangsu Academy of Agricultural Sciences/Jiangsu Key Laboratory for Horticultural Crop Genetic Improvement, 210014 Nanjing, China

\section{Author contributions}

C.G. and S.L.Z. conceived this study; C.G. conducted transcriptome-based data analyses, isolated the candidate genes by GRT-PCR, and drafted the paper. Z.H.G. contributed to most experiments with help from Y.J.Z. and Z.H.X. Y.Y.Z. 
collected peach samples at the developing and ripening stages. J.L.Y. and S.L.Z. revised this paper.

\section{Conflict of interest}

The authors declare no competing interests.

Supplementary information The online version contains supplementary material available at https://doi.org/10.1038/s41438-021-00644-6.

Received: 21 January 2021 Revised: 17 June 2021 Accepted: 14 July 2021 Published online: 01 October 2021

\section{References}

1. Klee, H. J. \& Giovannoni, J. J. Genetics and control of tomato fruit ripening and quality attributes. Annu. Rev. Genet. 45, 41-59 (2011).

2. Vrebalov, J. et al. A MADS-box gene necessary for fruit ripening at the tomato ripening-inhibitor (Rin) locus. Science 296, 343-346 (2002).

3. Pech, J. C., Purgatto, E., Bouzayen, M. \& Latché, A. Ethylene and fruit ripening Annu. Plant Rev. 44, 275-304 (2012).

4. Merchante, C. et al. Ethylene is involved in strawberry fruit ripening in an organ-specific manner. J. Exp. Bot. 64, 4421-4439 (2013).

5. Villarreal, N. M., Bustamante, C. A., Civello, P. M. \& Martínez, G. A. Effect of ethylene and 1-MCP treatments on strawberry fruit ripening. J. Sci. Food Agric. 90, 683-689 (2010).

6. Gu, C. et al. Multiple regulatory roles of AP2/ERF transcription factor in angiosperm. Bot. Stud. 58, 6 (2017).

7. Guo, H. W. \& Ecker, J. R. The ethylene signaling pathway: new insights. Curr. Opin. Plant Biol. 7, 40-49 (2004).

8. Seymour, G. B., Ostergaard, L., Chapman, N. H., Knapp, S. \& Martin, C. Fruit development and ripening. Annu. Rev. Plant Biol. 64, 219-241 (2013).

9. Li, Y. C. et al. LeERF1 positively modulated ethylene triple response on etiolated seedling, plant development and fruit ripening and softening in tomato. Plant Cell Rep. 26, 1999-2008 (2007).

10. Lee, J. M. et al. Combined transcriptome, genetic diversity and metabolite profiling in tomato fruit reveals that the ethylene response factor SIERF6 plays an important role in ripening and carotenoid accumulation. Plant J. 70, 191-204 (2012).

11. Xiao, Y. Y. et al. Banana ethylene response factors are involved in fruit ripening through their interactions with ethylene biosynthesis genes. J. Exp. Bot. 64, 2499-2510 (2013).

12. Li, T. et al. Apple (Malus domestica) MdERF2 negatively affects ethylene biosynthesis during fruit ripening by suppressing MdACS1 transcription. Plant J. 88, 735-748 (2016).

13. Hao, P. P. et al. Transcriptome analysis unravels an ethylene response factor involved in regulating fruit ripening in pear. Physiol. Plant. 163, 124-135 (2018).

14. Fu, C. C. et al. Papaya CpERF9 acts as a transcriptional repressor of cell-wallmodifying genes CPPME1/2 and CPPG5 involved in fruit ripening. Plant Cell Rep. 35, 2341-2352 (2016).

15. Li, T. et al. The jasmonate-activated transcription factor MdMYC2 regulates ETHYLENE RESPONSE FACTOR and ethylene biosynthetic genes to promote ethylene biosynthesis during apple fruit ripening. Plant Cell 29, 1316-1334 (2017).

16. Yue, P. et al. Auxin-activated MdARF5 induces the expression of ethylene biosynthesis genes to initiate apple fruit ripening. N. Phytol. 226, 1781-1795 (2020).

17. Breitel, D. A. et al. AUXIN RESPONSE FACTOR 2 intersects hormonal signals in the regulation of tomato fruit ripening. PLoS Genet. 12, e1005903 (2016).

18. Hao, Y. W. et al. Auxin response factor SIARF2 is an essential component of the regulatory mechanism controlling fruit ripening in tomato. PLOS Genet. 11, e1005649 (2015).

19. Karlova, R. et al. Transcriptional control of fleshy fruit development and ripening. J. Exp. Bot. 65, 4527-4541 (2014).

20. Weng, L. et al. The zinc finger transcription factor SIZFP2 negatively regulates abscisic acid biosynthesis and fruit ripening in tomato. Plant Physiol. 167, 931-949 (2015).

21. Gao, Y. et al. A NAC transcription factor, NOR-like1, is a new positive regulator of tomato fruit ripening. Hortic. Res. 5, 75 (2018).
22. Osorio, S. et al. Systems Biology of tomato fruit development: combined transcript, protein, and metabolite analysis of tomato transcription factor (nor, rin) and ethylene receptor $(\mathrm{Nr})$ mutants reveals novel regulatory interactions. Plant Physiol. 157, 405-425 (2011).

23. Zhu, M. et al. A new tomato NAC (NAM/ATAF1/2/CUC2) transcription factor, SINAC4, functions as a positive regulator of fruit ripening and carotenoid accumulation. Plant Cell Physiol. 55, 119-135 (2014).

24. Meng, C. et al. Suppression of tomato SINAC1 transcription factor delays fruit ripening. J. Plant Physiol. 193, 88-96 (2016).

25. Wang, W. Q. et al. Genome-wide analysis of coding and non-coding RNA reveals a conserved miR164-NAC regulatory pathway for fruit ripening. $N$. Phytol. 225, 1618-1634 (2019).

26. Shan, W. et al. Molecular characterization of banana NAC transcription factors and their interactions with ethylene signalling component EIL during fruit ripening. J. Exp. Bot. 63, 5171-5187 (2012).

27. Rios, P. et al. ETHQV6.3 is involved in melon climacteric fruit ripening and is encoded by a NAC domain transcription factor. Plant J. 91, 671-683 (2017).

28. Zhou, H. et al. Molecular genetics of blood-fleshed peach reveals activation of anthocyanin biosynthesis by NAC transcription factors. Plant J. 82, 105-121 (2015).

29. Fujisawa, M., Nakano, T., Shima, Y. \& Ito, Y. A large-scale identification of direct targets of the tomato MADS box transcription factor RIPENING INHIBITOR reveals the regulation of fruit ripening. Plant Cell 25, 371-386 (2013).

30. Fujisawa, $M$. et al. Transcriptional regulation of fruit ripening by tomato FRUITFULL homologs and associated MADS box proteins. Plant Cell 26, 89-101 (2014).

31. Hayama, H., Tatsuki, M., Ito, A. \& Kashimura, Y. Ethylene and fruit softening in the stony hard mutation in peach. Postharvest Biol. Technol. 41, 16-21 (2006).

32. Tonutti, P., Casson, P. \& Ramina, A. Ethylene biosynthesis during peach fruit development. J. Am. Soc. Hortic. Sci. 116, 274-279 (1991).

33. Hayama, H., Shimada, T., Fujii, H., Ito, A. \& Kashimura, Y. Ethylene-regulation of fruit softening and softening-related genes in peach. J. Exp. Bot. 57, 4071-4077 (2006).

34. Gu, C. et al. Copy number variation of a gene cluster encoding endopolygalacturonase mediates flesh texture and stone adhesion in peach. J. Exp. Bot. 67, 1993-2005 (2016).

35. Bregoli, A. M. et al. Peach (Prunus persica) fruit ripening: aminoethoxyvinylglycine (AVG) and exogenous polyamines affect ethylene emission and flesh firmness. Physiol. Plant. 114, 472-481 (2002).

36. Hayama, H., Tatsuki, M., Ito, A. \& Kashimura, Y. Combined treatment of aminoethoxyvinylglycine (AVG) and 1-methylcyclopropene (1-MCP) reduces melting-flesh peach fruit softening. Postharvest Biol. Technol. 50, 228-230 (2008).

37. Tadiello, A. et al. On the role of ethylene, auxin and a GOLVEN-like peptide hormone in the regulation of peach ripening. BMC Plant Biol. 16, 44 (2016).

38. Tatsuki, M., Haji, T. \& Yamaguchi, M. The involvement of 1-aminocyclopropane1- carboxylic acid synthase isogene, Pp-ACS1, in peach fruit softening. J. Exp. Bot. 57, 1281-1289 (2006). 2006.

39. Tonutti, P., Bonghi, C., Ruperti, B., Tornielli, G. B. \& Ramina, A. Ethylene evolution and 1-aminocyclopropane-1-carboxylate oxidase gene expression during early development and ripening of peach fruit. J. Am. Soc. Hortic. Sci. 122, 642-647 (1997). 1997.

40. Gu, C. et al. A HD-ZIP $\|$ HOMEOBOX transcription factor, PpHB.G7, mediates ethylene biosynthesis during fruit ripening in peach. Plant Sci. 278, 12-19 (2019).

41. Guo, Z. H. et al. Expression analysis of TCP genes in peach reveals an involvement of PPTCP.A2 in ethylene biosynthesis during fruit ripening. Plant Mol. Biol. Report. 36, 588-595 (2018).

42. $\mathrm{Ge}, \mathrm{H}$. et al. EjNAC3 transcriptionally regulates chilling-induced lignification of loquat fruit via physical interaction with an atypical CAD-like gene. J. Exp. Bot. 68, 5129-5136 (2017).

43. Nieuwenhuizen, N. J. et al. Natural variation in monoterpene synthesis in kiwifruit: transcriptional regulation of terpene synthases by NAC and ETHYLENE-INSENSITIVE3-like transcription factors. Plant Physiol. 167, 1243-1258 (2015).

44. Zhong, R. Q., Lee, C. H. \& Ye, Z. H. Global analysis of direct targets of secondary wall NAC master switches in arabidopsis. Mol. Plant 3, 1087-1103 (2010).

45. Nakano, T., Suzuki, K., Fujimura, T. \& Shinshi, H. Genome-wide analysis of the ERF gene family in Arabidopsis and rice. Plant Physiol. 140, 411-432 (2006).

46. Zhang, C. H. et al. Genome-wide analysis of the AP2/ERF superfamily in peach (Prunus persica). Genet. Mol. Res. 11, 4789-4809 (2012). 
47. Licausi, F., Ohme-Takagi, M. \& Perata, P. APETALA2/Ethylene Responsive Factor (AP2/ERF) transcription factors: mediators of stress responses and developmental programs. N. Phytol. 199, 639-649 (2013).

48. Trainotti, L., Tadiello, A. \& Casadoro, G. The involvement of auxin in the ripening of climacteric fruits comes of age: the hormone plays a role of its own and has an intense interplay with ethylene in ripening peaches. J. Exp. Bot. 58, 3299-3308 (2007).

49. Pan, L. et al. PpYUC11, a strong candidate gene for the stony hard phenotype in peach (Prunus persica L. Batsch), participates in IAA biosynthesis during fruit ripening. J. Exp. Bot. 66, 7031-7044 (2015).

50. Cirilli, M. et al. Integrative genomics approaches validate PpYUC11-like as candidate gene for the stony hard trait in peach ( $P$. persica $L$. Batsch). BMC Plant Biol. 18, 88 (2018).

51. Hu, R. et al. Comprehensive analysis of NAC domain transcription factor gene family in Populus trichocarpa. BMC Plant Biol. 10, 145 (2010).

52. Nuruzzaman, M. et al. Genome-wide analysis of NAC transcription factor family in rice. Gene 465, 30-44 (2010).

53. Jensen, M. K. \& Skriver, K. NAC transcription factor gene regulatory and protein-protein interaction networks in plant stress responses and senescence. IUBMB Life 66, 156-166 (2014).

54. Kim, H. J., Nam, H. G. \& Lim, P. O. Regulatory network of NAC transcription factors in leaf senescence. Curr. Opin. Plant Biol. 33, 48-56 (2016).

55. Olsen, A. N., Ernst, H. A., Lo Leggio, L. \& Skriver, K. NAC transcription factors: structurally distinct, functionally diverse. Trends Plant Sci. 10 79-87 (2005).

56. Zhong, R. Q., Demura, T. \& Ye, Z. H. SND1, a NAC domain transcription factor, is a key regulator of secondary wall synthesis in fibers of Arabidopsis. Plant Cell 18, 3158-3170 (2006).

57. Shang, X. G. et al. A cotton NAC transcription factor GhirNAC2 plays positive roles in drought tolerance via regulating ABA biosynthesis. Plant Sci. 296 110498 (2020)

58. Ren, T. T. et al. Involvement of NAC transcription factor SiNAC1 in a positive feedback loop via ABA biosynthesis and leaf senescence in foxtail millet. Planta 247, 53-68 (2018).

59. Mao, C. J. et al. A rice NAC transcription factor promotes leaf senescence via ABA biosynthesis. Plant Physiol. 174, 1747-1763 (2017).

60. Ma, X. M. et al. The NAC Transcription Factor SINAP2 Regulates Leaf Senescence and Fruit Yield in Tomato. Plant Physiol. 177, 1286-1302 (2018).

61. Zhang, M., Yuan, B. \& Leng, P. The role of ABA in triggering ethylene biosynthesis and ripening of tomato fruit. J. Exp. Bot. 60, 1579-1588 (2009).
62. Kou, X. H. et al. The interplay between ABA Vethylene and NAC TFs in tomato fruit ripening: a review. Plant Mol. Biol. 106, 223-238 (2021).

63. Shinozaki, Y. et al. High-resolution spatiotemporal transcriptome mapping of tomato fruit development and ripening. Nat. Commun. 9, 364 (2018).

64. Lang, Z. et al. Critical roles of DNA demethylation in the activation of ripeninginduced genes and inhibition of ripening-repressed genes in tomato fruit. Proc. Natl Acad. Sci. 114, E4511-E4519 (2017).

65. Liu, R. et al. A DEMETER-like DNA demethylase governs tomato fruit ripening. Proc. Natl Acad. Sci. 112, 10804-10809 (2015).

66. Gao, C. et al. MicroRNA profiling analysis throughout tomato fruit development and ripening reveals potential regulatory role of RIN on microRNAs accumulation. Plant Biotechnol. J. 13, 370-382 (2015).

67. Moxon, S. et al. Deep sequencing of tomato short RNAs identifies microRNAs targeting genes involved in fruit ripening. Genome Res. 18, 1602-1609 (2008).

68. Zhu, B. et al. RNA sequencing and functional analysis implicate the regulatory role of long non-coding RNAs in tomato fruit ripening. J. Exp. Bot. 66, 4483-4495 (2015)

69. Li, R., Fu, D., Zhu, B., Luo, Y. \& Zhu, H. CRISPR/Cas9-mediated mutagenesis of IncRNA1459 alters tomato fruit ripening. Plant J. 94, 513-524 (2018).

70. Lu, P. et al. Genome encode analyses reveal the basis of convergent evolution of fleshy fruit ripening. Nat. Plants 4, 784-791 (2018).

71. Gu, C. et al. RNA-Seq analysis unveils gene regulation of fruit size cooperatively determined by velocity and duration of fruit swelling in peach. Physiol. Plant. 164, 320-336 (2018).

72. Liu, Y. et al. A WRKY transcription factor PbrWRKY53 from Pyrus betulaefolia is involved in drought tolerance and AsA accumulation. Plant Biotechnol. J. 17, 1770-1787 (2019).

73. Fan, X., Argenta, L. \& Mattheis, J. P. Inhibition of ethylene action by 1-methylcyclopropene prolongs storage life of apricots. Postharvest Biol. Technol. 20, 135-142 (2000).

74. Han, Y. C. et al. Banana transcription factor MaERF11 recruits histone deacetylase MaHDA1 and represses the expression of MaACO1 and expansins during fruit ripening. Plant Physiol. 171, 1070-1084 (2016).

75. Yin, X. R. et al. Involvement of an ethylene response factor in chlorophyl degradation during citrus fruit degreening. Plant J. 86, 403-412 (2016).

76. Chen, J. et al. Phosphatidic Acid Counteracts S-RNase Signaling in Pollen by Stabilizing the Actin Cytoskeleton. Plant Cell 30, 1023-1039 (2018).

77. Sultana, A. \& Lee, J. E. Measuring protein-protein and protein-nucleic acid interactions by biolayer interferometry. Curr. Protoc. Protein Sci. 79 19.25.1-19.25.26 (2015). 